\title{
Texas healthcare worker is diagnosed with Ebola
}

\author{
Michael McCarthy \\ Seattle
}

Ebola disease has been diagnosed in a health worker in Texas, state health officials said Sunday 12 October. The worker, who press reports say is a nurse, took care of a Liberian man who fell ill shortly after arriving in the United States and later died of Ebola disease. ${ }^{1}$

The worker is the second person to contract the disease outside Africa. The first, Teresa Romero, is a Spanish nurse who cared for two Spanish missionaries who had been flown home from Africa after becoming ill. ${ }^{2}$

In a press briefing Sunday the director of the Centers for Disease Control and Prevention, Tom Frieden, said it was not known how the Texas health worker had become infected, "but at some point there was a breach in protocol and that breach in protocol resulted in this infection." The worker had "extensive contact" with the Liberian patient while he was in the hospital, Frieden said.

The patient, Thomas Eric Duncan, 42, flew from Liberia 19 September and arrived in Dallas the next day. He had no symptoms during his trip but fell ill shortly after arrival and on 25 September went to the emergency department of Texas Health Presbyterian Hospital Dallas, a large, acute care community hospital. There he was evaluated and discharged with antibiotics and a diagnosis of sinusitis.

Duncan returned, severely ill, on 28 September, and was admitted. Ebola disease was diagnosed 30 September. Despite ventilator support, renal dialysis, and treatment with an experimental antiviral, brincidofovir (an oral nucleotide analog that has been shown to be active against the virus in vitro), he died 8 October. The hospital has no high level bio-containment facilities for isolating patients with highly contagious diseases. All hospital workers who had had contact with Duncan have been monitoring themselves for symptoms or fever, health officials said, and on the night of Friday 10 October the nurse reported that she had developed low grade fever. She was admitted through the hospital's emergency room and put into isolation within 90 minutes. A blood test conducted in Texas the next day was positive for the Ebola virus. On Sunday the CDC confirmed the diagnosis.

"The level of her symptoms and indications from the test itself suggest that the level of virus that she had was low," Frieden said.

Frieden said that the $\mathrm{CDC}$ was investigating how the nurse may have become exposed. "The care of Ebola can be done safely, but it is hard to do it safely," he said. "It requires meticulous and scrupulous attention to infection control, and even a single inadvertent innocent slip can result in contamination."
Ebola disease is caused by a filovirus first discovered in Africa in 1976. Infection is thought to require direct contact with bodily fluids of a person who has developed symptoms or with contaminated objects such as syringes. On average, symptoms develop 8-10 days after exposure, but the incubation period can range from two to 21 days. There is no vaccine. Treatment is supportive.

Since the current outbreak began in Guinea in December last year more than 4000 people have died from the infection in west Africa, primarily in Guinea, Liberia, and Sierra Leone.

While the investigation is under way, Frieden said, the CDC is recommending that hospitals keep the number of workers who care for patients with suspected Ebola disease to a minimum and that procedures be limited to those essential for the patients' care. The CDC will review its recommendations concerning the personal protective gear that health workers should wear while caring for patients suspected of having been infected with the virus, Frieden added.

CDC investigators will be looking particularly closely at two procedures, Frieden said: the performance of kidney dialysis and respiratory intubation. "Both of those procedures may spread contaminated materials and are considered high risk procedures. They were undertaken on the index patient as a desperate measure to try to save his life," he said.

David Lakey, commissioner of the Texas Department of Health and Human Services, said that 48 people in the community who came into contact or may have come into contact with Duncan are being monitored daily for signs of infection and will be for the 21 days after their last contact. So far none has developed fever or symptoms, he said. One person who was in contact with the new case while she might have been contagious has been admitted to the hospital for observation.

"Unfortunately it is possible in the coming days that we will see additional cases of Ebola," Lakey said. "This is because the healthcare workers who cared for this individual may have had a breach of the same nature as the individual who appears now to have preliminary positive tests."

In Washington, DC, Barack Obama was briefed about the new case by the assistant to the president for homeland security and counterterrorism, Lisa Monaco. He later spoke with the secretary of health and human services, Sylvia Burwell. The White House reported that Obama directed Burwell and the CDC to investigate the apparent breach in infection controls in the Dallas hospital as expeditiously as possible, review the hospital's infection control procedures and use of personal protective 
equipment, and share lessons from the inquiry quickly and broadly.

1 McCarthy M. Liberian man being treated for Ebola in Texas dies. BMJ 2014;349:g6145
2 Gulland A. Spanish authorities investigate how nurse contracted Ebola. BMJ 2014;349:g6120.

Cite this as: BMJ 2014;349:g6200

(c) BMJ Publishing Group Ltd 2014 